\title{
Synergistic Effect Between Sulfate-reducing Bacteria and Pseudomonas Aeruginosa on Corrosion Behavior of Q235 Steel
}

\author{
Guangfeng Xi ${ }^{1}$, Xiaodong Zhao ${ }^{2, ~ *, ~ S h u a i ~ W a n g ~}{ }^{2}$,Jie Yang ${ }^{2, ~ *}$, Jie Sun ${ }^{2}$, Zhongyi An ${ }^{2}$, Yan $\mathrm{Li}^{3}$, \\ Xinlei $Q u^{4}$ \\ ${ }^{1}$ Shandong Special Equipment Inspection Institute Lute Inspection\&Testing Co. Ltd, Jinan 250100, \\ China \\ ${ }^{2}$ School of Ocean, Yantai University, Yantai 264005, China; \\ ${ }^{3}$ Qingdao Branch of Naval Aeronautical Engineering Academy, Qingdao 266041, China; \\ ${ }^{4}$ Shandong Nanshan Aluminum Co., Ltd., Yantai 265706, China \\ *E-mail: danielxdzhao@ aliyun.com; kittyangj@163.com
}

doi: $10.20964 / 2020.01 .38$

Received: 25 August 2019 / Accepted: 22 October 2019 / Published: 30 November 2019

\begin{abstract}
Microbial community in nature is a whole in which the multiple species interact and restrict each other, leading to the metal corrosion as a synergistic result of microorganisms. In this paper, the corrosion behavior of Q235 steel in the culture media containing pure sulfate-reducing bacteria, Pseudomonas aeruginosa and their mixed cultures were analyzed by electrochemical methods and stereoscopic microscope. The results showed that the steel were subjected to corrosion to different degrees after immersion in the three cultures and with different characteristics. The corrosion of Q235 steel was the most severe in the pure SRB-containing culture, and then followed by that in pure PAO-containing culture. The corrosion process of Q235 steel in the mixed cultures was more complicated. The corrosion was alleviated compared to that in pure strain system, indicating that the coexistence of mixed strains might change the effect on biocorrosion process.
\end{abstract}

Keywords: Microbiologically influenced corrosion (MIC); Pseudomonas aeruginosa; Sulfatereducing bacteria; Bacterial synergism

\section{FULL TEXT}

(C) 2020 The Authors. Published by ESG (www.electrochemsci.org). This article is an open access article distributed under the terms and conditions of the Creative Commons Attribution license (http://creativecommons.org/licenses/by/4.0/). 